\title{
DesarRollo de un modelo matemático no lineAl APLICANDO MÍNIMOS CUADRADOS EN UN SISTEMA DE NIVEL
}

\author{
Paula A. Ortiz ${ }^{1}$, \\ José L. RAMíREZ ${ }^{2}$, \\ Pedro L. Simanca ${ }^{3}$
}

\section{Resumen:}

Para efectuar el análisis de un sistema, es necesario obtener un modelo matemático que lo represente, con el cual se pueda simular la dinámica del proceso y desarrollar estrategias de control para luego ser aplicadas en el sistema real. Con este objetivo, es importante que el modelo, no siendo único, sea una representación fidedigna del proceso. En este trabajo se obtiene un modelo matemático no lineal para la planta de nivel ubicada en el laboratorio de medición y control de fluidos del INSTITUTO TECNOLÓGICO METROPOLITANO. La obtención del modelo consta de dos partes, la primera es encontrar las ecuaciones físicas del sistema en términos de algunos parámetros desconocidos, la segunda es encontrar estos parámetros usando mínimos cuadrados. Se realizó una validación del modelo obtenido sobre la planta y se encontró que el modelo era confiable. Esta técnica de identificación de sistemas puede ser aplicada a otros procesos, en

1 Ingeniera en Instrumentación y Control. Magister en Ingeniería área Automática. Docente de tiempo completo Instituto Tecnológico Metropolitano, paulaortiz@ itm.edu.co

2 Ingeniero eléctrico: Magister en Ingeniería área Automática. Decano Facultad de Ingenierías Instituto Tecnológico Metropolitano, joseramirez@itm.edu.co

3 Ingeniero Mecánico: Magister en sistemas automáticos de producción. Decano Facultad de Ciencias Instituto Tecnológico Metropolitano, pedrosimanca@itm. edu.co 
los cuales se conozcan las ecuaciones físicas del sistema en términos de parámetros desconocidos.

\section{Palaloras clave:}

Identificación de sistemas, sistema no lineal, mínimos cuadrados, modelo matemático, proceso dinámico

\section{Alostract:}

To perform a system analysis it is necessary to have a mathematical model representing it, which permits simulation of the process dynamics and development of control strategies to be applied in the real system. With this aim, it is important for the model, not being unique, to be a close representation of the process. On this work a non lineal mathematical model is obtained for the level plant at the laboratory of control and measurement of fluidics of the INSTITUTO TECNOLÓGICO METROPOLITANO. Obtaining the model consists of two parts, the first one consists in finding physical equations of the system in terms of some unknown parameters, and the second one consists in finding those parameters using least square method. A validation of the obtained model was made and it was found that the model was reliable. This system identification technique can be applied in other process with known physical equations in terms of unknown parameters.

\section{Keywords:}

Systems identification, nonlinear system, minimal square, mathematical model, dynamic process. 


\section{INTRODUCGIÓN}

La obtención de un modelo matemático consta de dos partes. La primera es obtener las ecuaciones dinámicas que rigen el comportamiento del sistema a partir de ecuaciones físicas. De esta manera, se tiene la estructura del modelo matemático, en términos de algunos parámetros que varían según las características del sistema que se desean modelar, entonces, la segunda parte es obtener el valor de estos parámetros utilizando alguna técnica de identificación, en este caso se identifica el proceso con el método de mínimos cuadrados. Además, el proceso de modelado es frecuentemente iterativo; un modelo matemático basado en las leyes físicas sugerirá cuales datos experimentales deben tomarse y a su vez el modelo puede llevar grandes refinamientos con el fin de acoplarse a los datos reales.

La primera parte del problema tiene amplia cobertura en la literatura, de manera que la estructura de los modelos matemáticos para una planta de nivel es conocida, entonces el problema central está en la identificación de los parámetros desconocidos. A continuación se presenta una revisión de algunos artículos que explican el modelo matemático.

En el trabajo de (Ríos \& Toro, 2006), se explica la estimación de parámetros en modelos ARMA (Autoregressive, Moving Average). Estos modelos son basados en la representación de planta, que consiste en un formato en el cual la salida $y(t)$ actual se expresa como una función lineal de salidas pasadas y(t-j) y de las entradas pasadas $\mathrm{u}(\mathrm{t}-\mathrm{j}-\mathrm{d})$, donde $\mathrm{d}$ es el retardo. Los sistemas son tratados como cajas negras donde se tiene una señal de entrada, una de salida y las perturbaciones que afectan al sistema, este modelo da una representación lineal del sistema identificado en un punto de operación, donde se estima el modelo solo con datos experimentales, no se conoce las ecuaciones matemáticas que rigen al proceso.

(Barajas, 2004) presentaron un estudio sobre la Identificación de parámetros de un modelo matemático mediante un algoritmo basado en cuasi-linearización y mínimos cuadrados, demostrando 
que el método de identificación de parámetros, basados en la cuasi-linearización y mínimos cuadrados es útil para determinar los parámetros desconocidos de un sistema dinámico lineal o no-lineal. Para la obtención de los parámetros del modelo, se requerirá del modelo matemático del sistema, las trayectorias de las señales de entrada y el comportamiento del sistema durante un estado transitorio en el que todos sus modos o elementos sean excitados. Para determinar los parámetros desconocidos se presentará un índice de comportamiento, el mismo que será minimizado, de tal manera que las trayectorias del modelo simulen exactamente las trayectorias del sistema real. Obteniéndose del proceso los valores de los parámetros desconocidos, este modelo es aceptable para una zona de trabajo que es donde se toman los datos.

(Durango et al., 2004), desarrollan un Modelo matemático para secador de alimentos de flujo radial, la metodología siguió el diseño de un experimento factorial, el cual, mediante análisis estadístico, posibilitó la obtención del modelo matemático que describe el comportamiento del proceso para un tiempo de secado de tres horas. Las variable estudiadas fueron: relación superficie/ volumen, masa de yuca, temperatura del aire de recirculación y velocidad de flujo de aire. El análisis estableció que las variables de mayor influencia en el proceso son la relación superficie/volumen, seguida por la masa de material que se iba a secar.

La obtención de un modelo matemático es necesaria en cualquier proceso que se lleve a cabo para analizar el comportamiento del sistema. Aunque el modelo matemático no es único, se debe garantizar que sea confiable. De acuerdo a esto, en este trabajo se desarrolla el modelo matemático a partir de leyes físicas, las cuales se encuentran en términos de parámetros dinámicos que varían con los cambios de la altura en los tanques, los flujos de entrada y salida y la señal de control, los cuales son calculados usando identificación por el método de mínimos cuadrados. 


\section{Materiales y métodos}

\subsection{Descripción de la Planta de nivel}

\section{El INSTITUTO TECNOLÓGICO METROPOLITANO} cuenta con un laboratorio de medición y control de fluidos. En la Fig. 1 se presenta el diagrama de instrumentos para el sistema de nivel, el cual cumple las normas ISO (ISO4006, 1991).

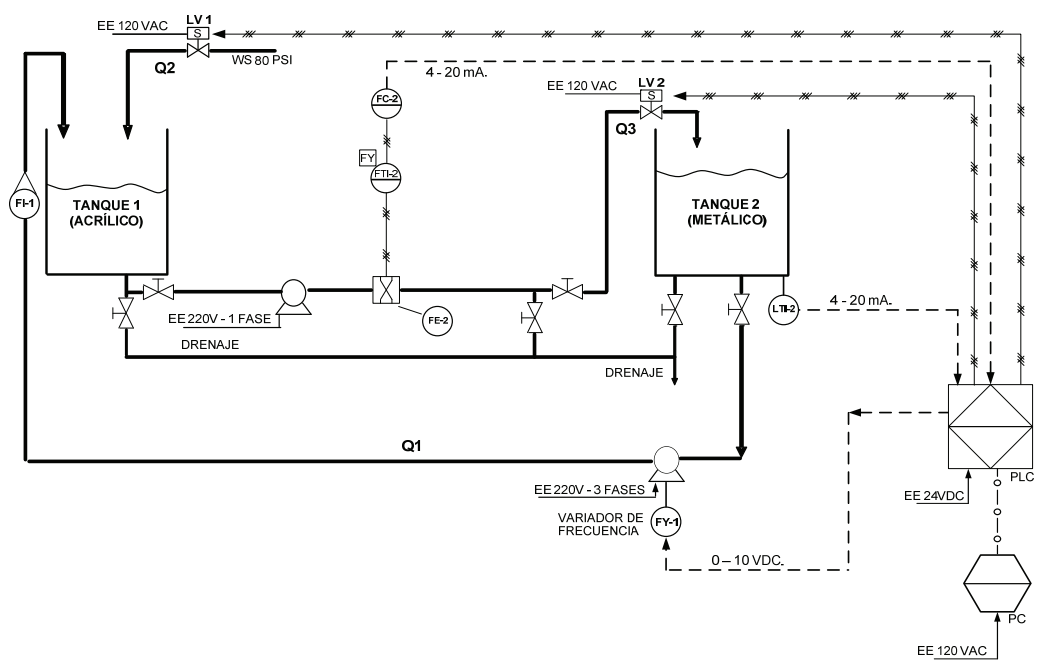

Fig. 1. Diagrama de InStRumentos PARA EL CONTROL DE NIVEL

Los equipos con los que cuenta la planta son: Un tanque metálico construido en lámina de acero SAE 1020 de 3/16" de espesor, con un diámetro de $98 \mathrm{~cm}$ y una altura de $115 \mathrm{~cm}$; un tanque de acrílico construido en lámina de acrílico transparente de $10 \mathrm{~mm}$ de espesor, tiene un diámetro de $79 \mathrm{~cm}$ y una altura de $98 \mathrm{~cm}$; un variador de velocidad, el cual se muestra en la Fig. 2, cuya función es la de controlar la velocidad del motor trifásico para accionar una bomba centrífuga (Ordóñez, 2005) 


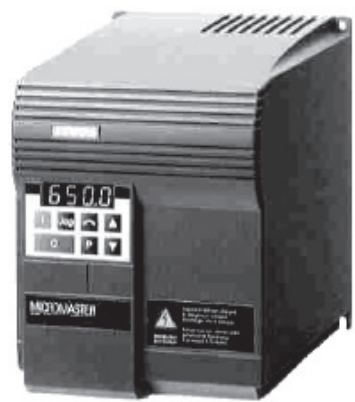

Fig. 2. Variador de Velocidad (Siemens, 2002)

Un transmisor Sitrans P, el cual se muestra en la Fig. 3, este transmisor de presión diferencial se encuentra localizado entre el tanque de acrílico y el de metal, el cual envía una señal de 4 a 20 $\mathrm{mA}$ al PLC, proporcional al caudal que llega al tanque de acrílico, gracias a la diferencia de presiones a la entrada y a la salida del transmisor ocasionada por la placa de orificio. (Rosemount, 2005).

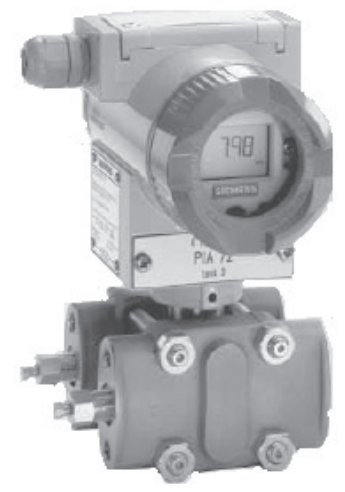

Fig. 3. TRANSmisor de FLUJo

Un transmisor de presión diferencial (LTI - 2), (Ramírez, 2007), para la medición de nivel de agua, el cual se muestra en la Fig. 4. Está instalado en el tanque de metal. Este transmisor envía una señal de 4 a $20 \mathrm{~mA}$ al controlador lógico programable (PLC), reportando la presión actual del tanque, dato que se puede convertir en medida de nivel. 


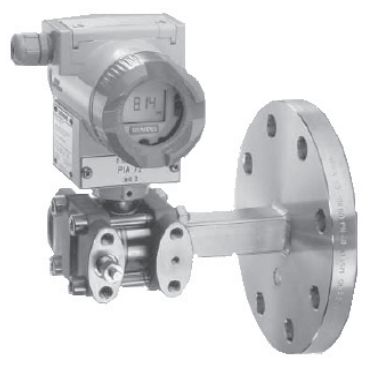

FIG. 4. TRANSmISOR DE NIVEL

Un panel táctil SIMATIC TP 270 de 10.4", color (256 colores), tienen todos los puertos integrados MPI, PROFIBUS DP, USB y Ethernet, y sirven para todo tipo de aplicaciones con manejo y visualización local de máquinas e instalaciones, tanto en la industria manufacturera como en la industria de procesos, al igual que en la automatización. Un comunicador de campo el cual permite ser utilizado sobre dispositivos para buses de campo HART o FOUNDATION FIELDBUS y una Bomba centrífuga trifásica (B2).

\subsection{Identificación paramétrica por el método de mínimos cuadrados}

La identificación de un sistema dinámico tiene por objeto la obtención del modelo matemático lineal o no lineal del sistema a partir de datos experimentales. En la Fig. 5, se muestra como el algoritmo de identificación construye un modelo matemático del sistema a partir de parejas de datos experimentales $\left(u_{i}, y_{i}\right)$ en cada instante de tiempo, donde $u_{i}$ es la entrada $y_{i}$ la correspondiente salida del sistema. 


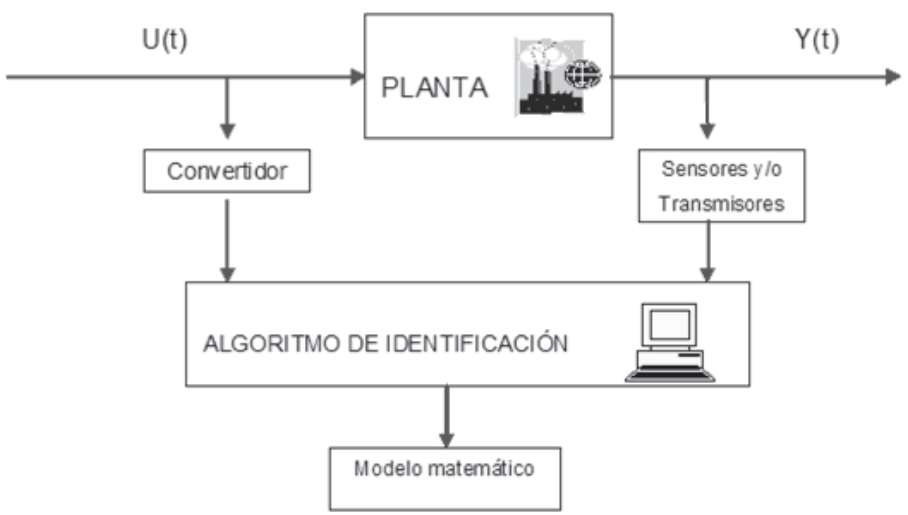

Fig. 5. PROCESO DE IDENTIFICACIÓN

Para la identificación de los parámetros desconocidos se usó una identificación paramétrica off-line por el método de mínimos cuadrado (He \& Asada, 1993). El criterio de mínimos cuadrados permite valorar el desempeño del modelo matemático enfrentado con cada dato medido, a partir de una función de costo J. El que arroje el menor valor numérico J ese es el mejor modelo matemático que representa el proceso (Ljung, 1991).

La función de costo $\mathrm{J}$ se puede hallar a partir de las siguientes, dependiendo de si se trata de un modelo continuo o discreto:

$J_{1}=\frac{1}{2} \int e^{2}(t) d t$, para modelos continuos

$J_{2}=\frac{1}{2} \sum e^{2}(k)$, para modelos discretos

En general:

$$
\begin{aligned}
& J(\theta)=\frac{1}{2} \sum_{k=0}^{n} f(e(k T, \theta)) \\
& e(k T)=y(k T)-\hat{y}(k T) \\
& f(e(k T, \theta))=e^{2}(k T)=(y(k T)-\hat{y}(k T))^{2}
\end{aligned}
$$


Donde:

$\theta$ son las variables estimados por el método de mínimos cuadrados. $T$ es el período de muestreo

$e$ es el error residual, definido por:

$$
e=\underset{\substack{\text { Respuesta } \\
\text { real }}}{y}-\underset{\begin{array}{c}
\text { Respuesta } \\
\text { estimada }
\end{array}}{\hat{y}}
$$

Para determinar si un modelo es mejor que otro, se compara el valor de $J$ obtenido con cada uno, siendo mejor aquel que implique el valor de función de costo $(J)$ más pequeño.

\subsection{Metodología propuesta}

Para el desarrollo del trabajo se siguen los siguientes pasos: primero se modela el sistema a partir de leyes físicas, una vez se tiene el modelo se determinan los parámetros desconocidos y la dependencia de estos con las variables del proceso; luego se toman los datos, para ello se debe garantizar la calibración de los instrumentos; una vez se toman los datos se realiza la identificación de los parámetros desconocidos por el método de mínimos cuadrados. Los datos que arroja el método de mínimos cuadrados deben de ser validados, para ello se toman nuevamente datos con una señal de excitación diferente a los datos tomados anteriormente, se compara estos datos con el modelo y se calcula el índice de desempeño, el cual dirá si el modelo es confiable o hay que volver a estimar los parámetros. El procedimiento descrito se muestra en la Fig. 6. 


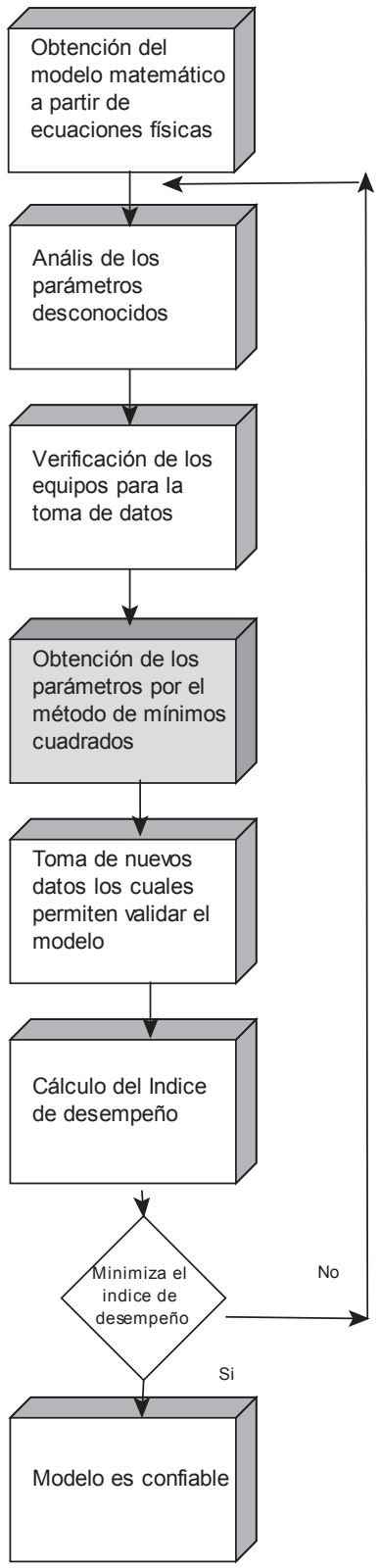

Fig. 6. Metodología propuesta 


\section{Resultados y discusión}

\subsection{Modelo Matemático}

Para desarrollar el modelo matemático del sistema, se parte del hecho de que el sistema cuenta con dos tanques que se encuentran conectados en paralelo, y está dotado de algunos equipos, los cuales se explicaron en el numeral anterior. El área de la superficie transversal de un tanque de sección circular, se puede calcular a partir de (7):

$$
A=\pi r^{2}
$$

Conociendo los valores de las áreas superficiales de los dos tanques, se plantean las ecuaciones de balance de masa en cada uno de los tanques, donde se considera que la densidad es constante, dando como resultado (8) y (9):

$$
\begin{gathered}
A_{1} \frac{d h_{1}}{d t}=Q_{1}+Q_{2}-Q_{3} \\
A_{2} \frac{d h_{2}}{d t}=Q_{3}-Q_{1}
\end{gathered}
$$

Donde

$Q_{1}$ es el caudal desde el tanque metálico al tanque acrílico

$Q_{2}$ es el caudal entrante al tanque acrílico a través de la electroválvula $Q_{3}$ es el caudal desde el tanque acrílico al tanque metálico

La ecuación (8) manifiesta que el cambio en la cantidad de agua contenida en el tanque acrílico es igual al caudal que entra desde el tanque metálico, más el caudal que entra desde la electroválvula, menos el caudal que fluye hacia el tanque metálico. De manera similar, (9) indica que el cambio en la cantidad de agua contenida en el tanque metálico es igual al caudal que entra desde el tanque acrílico menos el caudal que fluye hacia el tanque acrílico. 
Seguidamente, se hará uso de la ecuación de Torricelli para continuar con el desarrollo del modelo. La ecuación de Torricelli (Shames, 1998) se usa para calcular la velocidad de un líquido que sale a través de una apertura que se encuentra a cierta profundidad medida desde la superficie del líquido. La velocidad es directamente proporcional a la profundidad, debido a que a mayor profundidad, aumenta la presión y por lo tanto la fuerza con la que sale el líquido es mayor. Según la ecuación de Torricelli, la velocidad con la que sale el líquido por una abertura en el fondo del tanque es igual a:

$$
v=\sqrt{2 g h}
$$

Donde

$g=9.81 \mathrm{~m} / \mathrm{s}^{2}$ es la fuerza de gravedad

$h$ es la altura del tanque en $m$

Para encontrar el caudal que va desde el tanque metálico hacia el tanque acrílico $\left(Q_{1}\right)$, el caudal que entra al tanque acrílico a través de la electroválvula $\left(Q_{2}\right)$ y el caudal que va desde el tanque acrílico hacia el tanque metálico $\left(Q_{3}\right)$ se comenzó planteando (11)

$$
Q=K S v
$$

Donde:

$K$ es el factor que tiene en cuenta la estricción que sufre el fluido en su salida debido a la tubería, la válvula y la bomba. Este valor se determinó experimentalmente.

$S$ es el área orificio de salida.

Reemplazando (10) en (11), se tienen las siguientes expresiones para $Q_{1}$ y $Q_{3}$ :

$$
\begin{aligned}
& Q_{1}=u K_{1} S_{2} \sqrt{2 g h_{2}} \\
& Q_{3}=K_{2} S_{1} \sqrt{2 g h_{1}}
\end{aligned}
$$


donde

$u$ es la señal de control proveniente del variador de velocidad

Aunque el sistema cuenta con dos bombas, solo se ejerce acción de control sobre la bomba que lleva el caudal del tanque 2 al tanque 1, la bomba del tanque 1 al tanque 2 es de apertura fija, es por esto que solo se tiene en cuenta la variable $u$ en (12). Finalmente, si se reemplazan (12) y (13) en (8) y (9), se obtienen las siguientes expresiones:

$$
\begin{aligned}
& A_{1} \frac{d h_{1}}{d t}=u K_{1} S_{2} \sqrt{2 g h_{2}}+Q_{2}-K_{2} S_{1} \sqrt{2 g h_{1}} \\
& A_{2} \frac{d h_{2}}{d t}=K_{2} S_{1} \sqrt{2 g h_{1}}-u K_{1} S_{2} \sqrt{2 g h_{2}}
\end{aligned}
$$

Donde $K_{1}$ y $K_{2}$ son parámetros desconocidos que se encuentran experimentalmente, y es el factor que tiene en cuenta la estricción que sufre el fluido en su salida debido a la tubería, la válvula y la bomba.

\subsection{Toma de datos}

Para la obtención de los datos se determinaron las variables que se querían identificar y se verificó el estado de los equipos de recolección de datos experimentales, entre ellos: sensores, transmisores, elementos finales de control, P.C., software, fuentes de suministro eléctrico y neumático. El flujo de entrada al tanque $T 2$ proviene del tanque $T 1$ impulsado por la bomba monofásica $B 1$, la electroválvula $E V 2$ debe estar abierta en todo momento. El flujo de entrada es constante porque la bomba $B 1$ tiene velocidad constante y no se puede variar, el flujo de salida es manejado con la motobomba $B 2$ la cual es el elemento al que se le realizaron los cambios en la señal de entrada, modificándole la frecuencia. Cumplido los requerimientos se aplico la señal de excitación sobre la variable manipulada, como se muestra en la Fig. 7. 


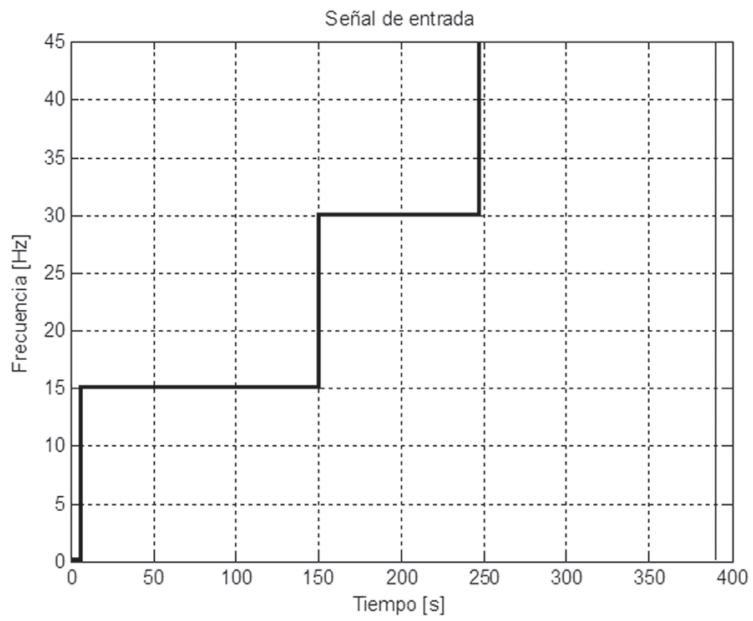

Fig. 7. Señal aplicada A la VARIable manipulada (Variador de Velocidad)

Luego, se tomaron los datos para cada lazo de control, aplicando cambios en la señal tipo escalón. Las curvas obtenidas para el nivel de los tanques 1 y 2 se muestran en las Fig. 8 y 9 , respectivamente. Las curvas obtenidas para los caudales $Q_{1}$ y $Q_{3}$ se muestran en las Fig. 10 y 11, respectivamente.

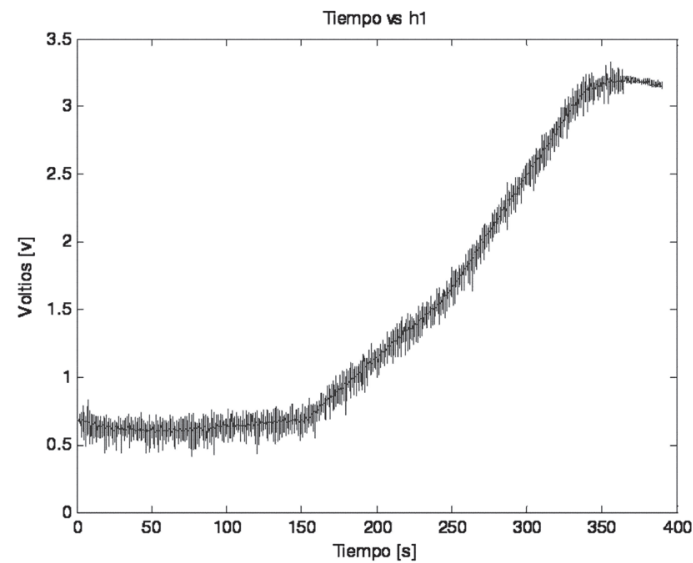

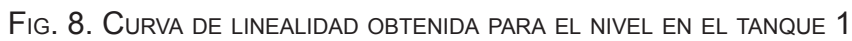
(TANQUE DE ACRILICO) 


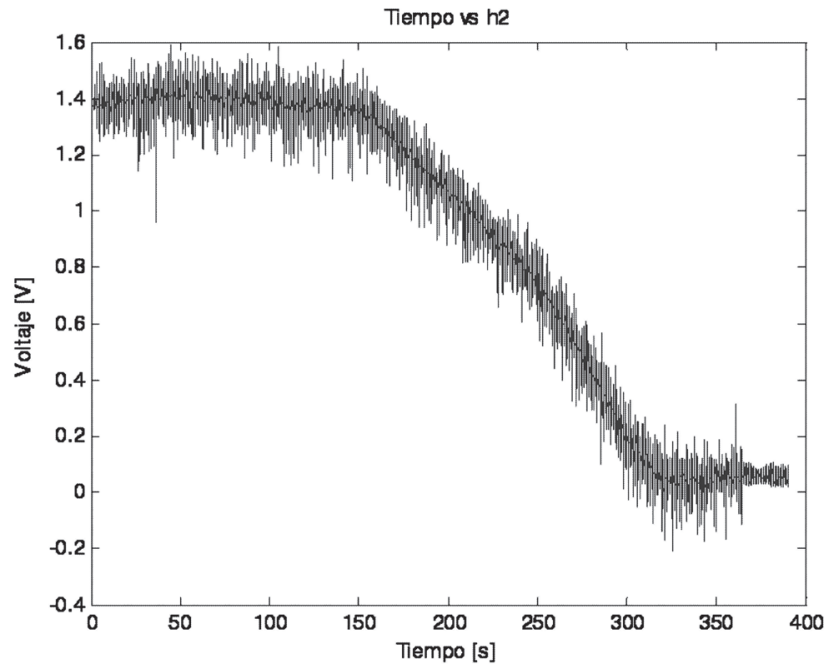

Fig. 9. Curva de linealidad obtenida para el nivel en el tandue 2 (TANQUE METÁLICO)

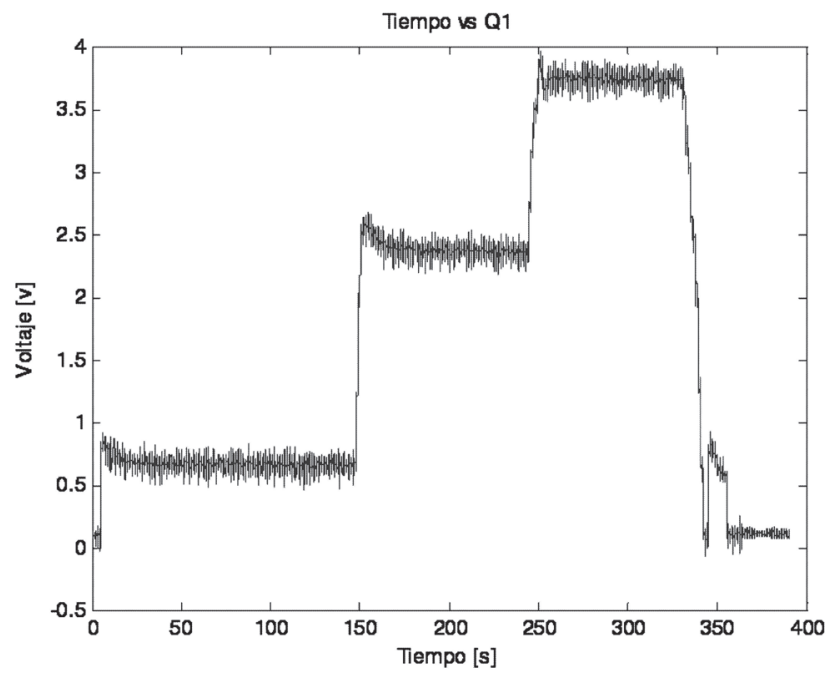

Fig. 10. CurVA DE LINEALIDAD obtenida PARA EL CAUdAL $Q_{1}$ (DESDE EL TANQUE METÁLICO AL TANQUE ACRÍLICO) 


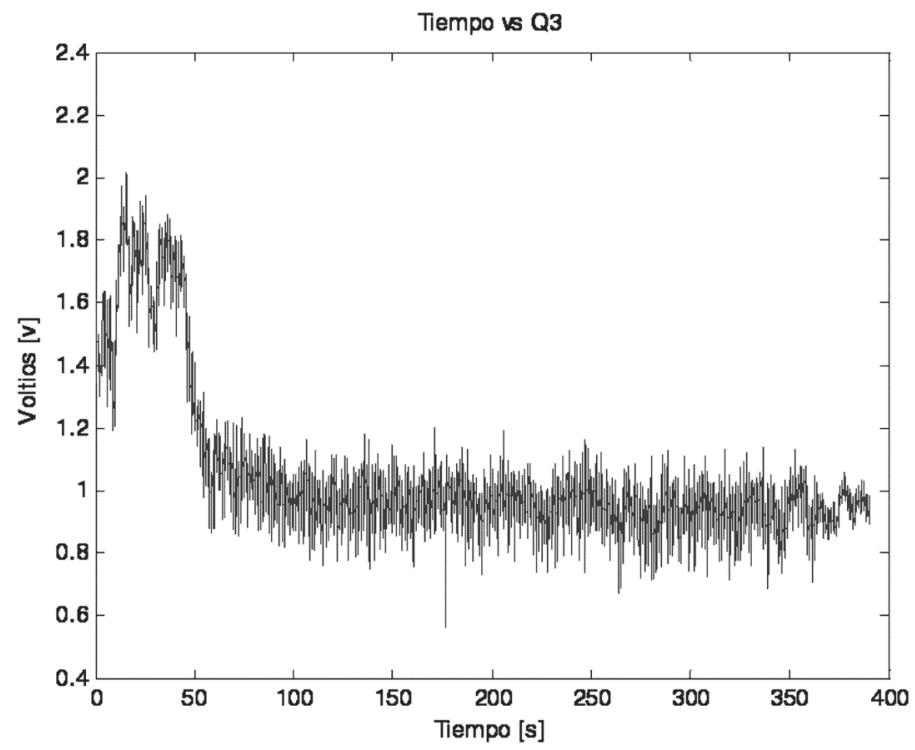

Fig. 11. CuRVA DE LINEALIDAD OBTENIDA PARA EL CAUDAL $Q_{3}$ (DESDE EL TANQUE ACRÍLICO AL TANQUE METÁLICO)

Con la información obtenida en las curvas de linealidad mostradas en las figuras anteriores, se desarrolló un programa en Matlab, cuya función es calcular los parámetros $K_{1}$ y $K_{2}$ usando (12) y (13).

\subsection{Identificación de los coeficientes $\boldsymbol{K}_{1} \mathbf{Y} \boldsymbol{K}_{2}$}

Para aplicar el método de mínimos cuadrados en la identificación del sistema, se define primero un conjunto de ecuaciones que permitan calcular los valores de $K_{1}$ y $K_{2}$ a partir de (12) se determina la dependencia de $K_{1}$ con cada una de las variables que interviene en el sistema, la cual depende la altura del tanque 2, el flujo 1 y la entrada de control y de (13) se determina la dependencia de $K_{2}$ con cada una de las variables que interviene en el sistema, la cual depende de la altura del tanque 1 , y el flujo 3, dando como resultado (16) y (17): 


$$
\begin{aligned}
& K_{1}=K_{10}+K_{1_{h_{2}}}\left(h_{2}\right)+K_{1_{Q_{1}}}\left(Q_{1}\right)+K_{1_{u}}(u), \\
& K_{2}=K_{20}+K_{2_{h_{1}}}\left(h_{1}\right)+K_{2_{Q_{3}}}\left(Q_{3}\right),
\end{aligned}
$$

Cada uno de los coeficientes que aparecen en (16) y (17) se identificará con el método de mínimos cuadrados. A continuación se explican los pasos para llevar a cabo la identificación. Primero se definen las matrices de estado

$$
\begin{gathered}
X\left(K_{1}\right)=\left[\begin{array}{cccc}
1 & h_{2}(1) & Q_{1}(1) & u(1) \\
1 & h_{2}(2) & Q_{1}(2) & u(2) \\
\vdots & \vdots & \vdots & \vdots \\
1 & h_{2}(n) & Q_{1}(n) & u(n)
\end{array}\right] \\
X\left(K_{2}\right)=\left[\begin{array}{ccc}
1 & h_{1}(1) & Q_{3}(1) \\
1 & h_{1}(2) & Q_{3}(2) \\
\vdots & \vdots & \vdots \\
1 & h_{1}(n) & Q_{3}(n)
\end{array}\right]
\end{gathered}
$$

Luego se define el vector de parámetros a estimar

$$
\begin{gathered}
K_{1}=\left[\begin{array}{lllll}
K_{0} & K_{1_{h_{2}}}\left(h_{2}\right) & K_{1_{Q_{1}}}\left(Q_{1}\right) & K_{1_{u}}(u)
\end{array}\right] \\
K_{2}=\left[\begin{array}{llll}
K_{0} & K_{2_{h_{1}}}\left(h_{1}\right) & K_{2_{Q_{3}}}\left(Q_{3}\right)
\end{array}\right]
\end{gathered}
$$

Se calculan los parámetros a estimar usando las siguientes ecuaciones

$$
\begin{aligned}
& \hat{\theta}_{1}=\left(X^{T} X\right)^{-1} X^{T} z_{1} \\
& \hat{\theta}_{2}=\left(X^{T} X\right)^{-1} X^{T} z_{2}
\end{aligned}
$$

Donde

$\mathrm{z}_{1}$ : Es el vector de los coeficientes de $K_{1}$, los cuales se obtiene al reemplazar los datos sensados en (12). 
$\mathrm{z}_{2}$ : Es el vector de los coeficientes de $K_{2}$, los cuales se obtiene al reemplazar los datos sensados en (13). El resultado obtenido con el método de identificación se muestra en (21):

$$
\begin{gathered}
\mathrm{k}_{1}=0,0006-0,0008 \mathrm{~h}_{2}+0,5087 \mathrm{Q}_{1} \\
\mathrm{k}_{2}=0,0037-0,03461 \mathrm{~h}_{1}+25,9234 \mathrm{Q}_{3}
\end{gathered}
$$

\subsection{Validación del método de identificación}

Para la verificación del modelo matemático se toman nuevamente datos de la planta, los cuales son comparados con el modelo obtenido. Esta comparación se realizó gráficamente, obteniéndose los resultados mostrados en las Fig. 12 y 13 . El valor estimado se halló a partir de las ecuaciones para $\hat{\theta}_{1}$ y $\hat{\theta}_{2}$ que se dieron en (20), multiplicados por $X$. El valor real se obtuvo a partir de mediciones sobre la planta. El indice de desempeño para $K_{1}$ fue de 0,02 y para $K 2$ fue de 0,09. Con lo cual se dice que el modelo es confiable.

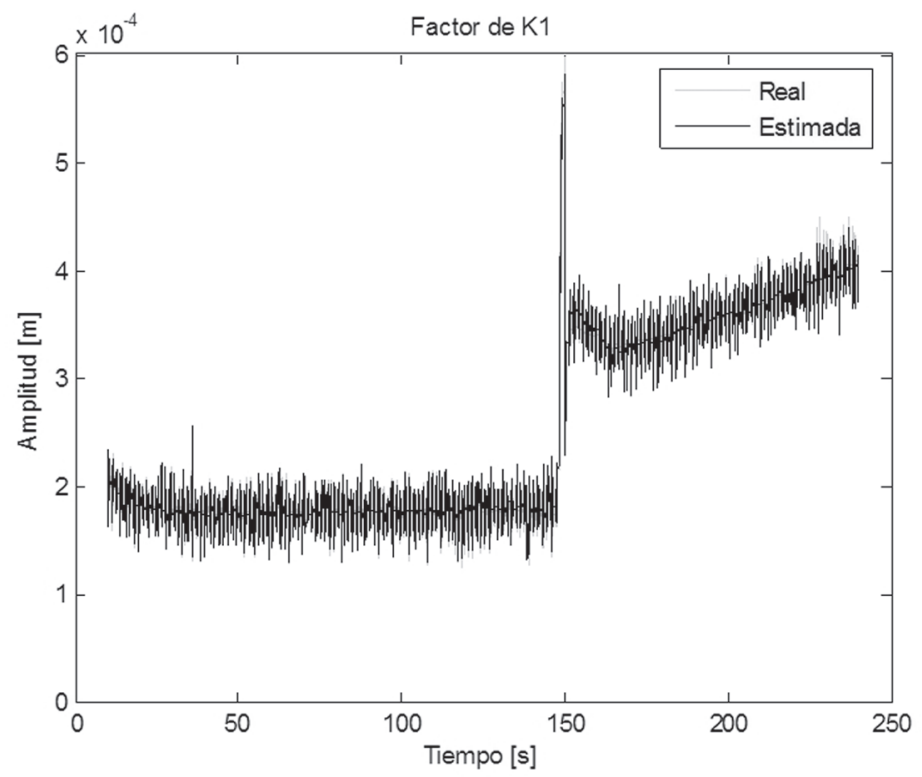

FIG. 12. Comparación ENTRE EL VALOR REAL Y ESTIMADO PARA K1 


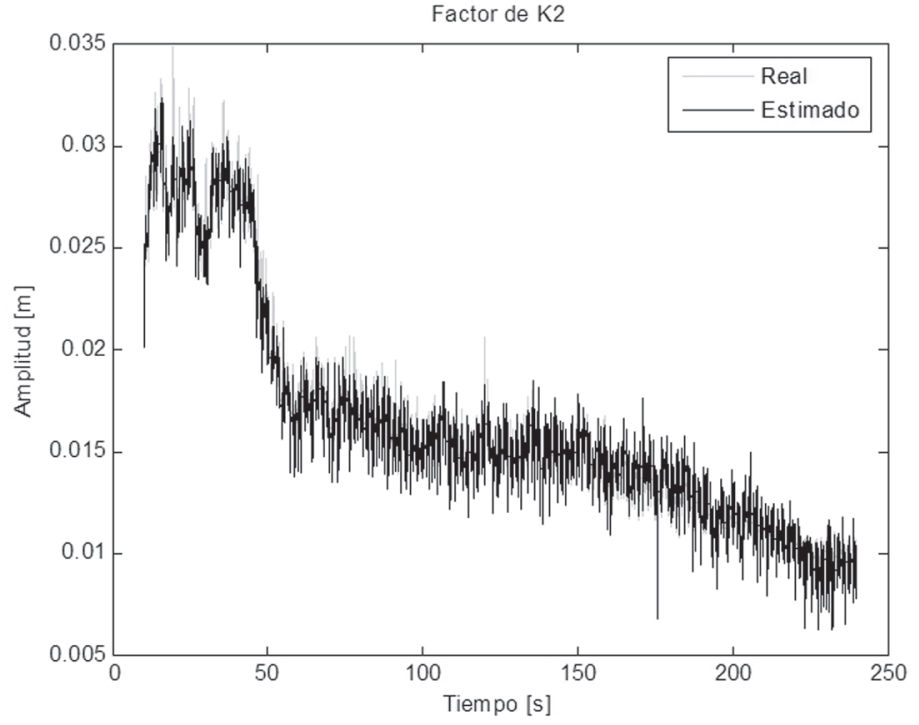

Fig. 13. Comparación ENTRE EL VALOR REAL Y EStimado PARA K2

\section{Conclusiones}

Una de las etapas más críticas del proyecto fue la selección de la instrumentación, de tal forma que se ajustara a los rangos de operación de la planta existente y que cumpliera la doble función de servir a las prácticas académicas y a las aplicaciones reales que se presentan en la industria. Sin embargo, esta tarea se llevó a cabo con éxito y se realizó en los equipos nuevos y en los ya existentes un proceso de ajuste y calibración que permitiera la confiabilidad en los resultados de las pruebas realizadas. Este proyecto es un prototipo a mediana escala, que cumple con las especificaciones técnicas de un sistema de nivel aplicado al sector productivo.

La identificación realizada con mínimos cuadrados permitió estimar los parámetros desconocidos para el sistema de nivel. La identificación de sistemas se realizó en lazo abierto, para evitar compensaciones del sistema en lazo cerrado. La identificación off-line permite obtener una idea básica del modelo, se recomienda 
una identificación on-line, que tome como base el modelo obtenido pero ajuste los parámetros dependiendo del punto de operación del sistema.

\section{Referencias}

Alonso, R., (2007); Comparative Analysis in Simulation Controlled Condition of a Fuzzy Adaptative PID Controller and a Clasic PID Acid - BaseNeutralizing Process Simulated on MATLAB Simulink, México, Universidad Tecnológica del Centro.

Barajas, L.E., (2004); Identificación de parámetros de un modelo matemático mediante un algoritmo basado en cuasi-linearización y mínimos cuadrados. Disponible en: http://biee.epn.edu.ec/dspace/handle/123456789/377

Durango, N., Bula A., Donaldo, L., (2004); Modelo matemático para secador de alimentos de flujo radial. Ingeniería y desarrollo: revista de la División de Ingeniería de la Universidad del Norte, (15), 1-8.

He X., Asada, H., (1993); A New Method for Identifying Orders of InputOutput. Models for Nonlinear Dynamic Systems, Proc. of the American Control Conf. S.F. California. [en línea]

http://www.math.ku.edu/ talata/documents/LD_Steel_Converter.pdf (Fecha De consulta: Diciembre de 2008)

ISO4006, (1991); Measurement of fluid flow in closed conduits; vocabulary and symbols.

Jantzen, J., (2003); Tuning Of Fuzzy PID Controllers, [En Línea]<http:// www.iau.dtu.dk/ jj/pubs/fpid.pdf> [Fecha de consulta Mayo de 2008].

Ljung, L., (1991); System Identification Toolbox User's Guide. [En Línea]. S.l: The MathWorks.

http://www.mathworks.com/access/helpdesk/help/pdf_doc/ident/ident.pdf> [fecha de consulta 15 de Julio de 2008].

Ordóñez, J.A., Trejos, D.A., (2005); Medidores de flujo, Mecánica de fluidos e hidráulica de tuberías. S.l: Universidad Francisco de Paula Santander. Facultad de ingeniería civil.

Ramírez, J.L., Londoño, C.M., (2007); Rediseño e implementación de un prototipo para la medición de nivel y caudal en el laboratorio de control de fluidos del ITM. Medellín, Trabajo de grado (Especialización Prentice Hall en Automática), Universidad Pontificia Bolivariana, Escuela de Ingenierías. 
Ríos, L.C., Toro, N., (2006); Estimación de parámetros en modelos arma por el criterio de mínimos cuadrados, Scientia et Technica, XII(31).

Rosemount, (2001); Manual de servicio del comunicador de campo 375. [En línea] http://www.emersonprocess.com/Rosemount/document/qig/008250109-4802.pdf [Fecha de consulta Junio de 2008]

Shames, I.H., (1998); Mecánica de Fluidos. Bogotá: McGraw- Hill.

Siemens, (1995); Catálogos SITRANS P Transmitter, HK series, ES series (Smart), Transmisores de Nivel. 7MF4622, Transmisores de Presión 7MF4022, Transmisores de Presión Diferencial y flujo 7MF4422, Operating Instrutructions, Orden No.: C73000-B5676-C90-2, Siemens AG. Bib.Personal.

Siemens, (2002); Manual Micromaster 440. Ed. 12/02. Erlangen: Siemens, 286p. (6SE6400-5BB00-0EP0).

Siemens, (1984); Catálogos MICROMASTER, Operating Instructions, Orden No.:G85139-H1750-U049-B. Erlangen: Siemens Plc. Bib.Personal, 2007. THE INTERNATIONAL SOCIETY FOR MEASUREMENT AND CONTROL. American National Standard, Instrumentation Symbols and Identification. North Carolina: ANSI/ISA, 122p. (ANSI/ISA S5.1).

Villanueva, C., Soto, R., (2007); Sistema de Control Difuso Para Nivel 1 de Temperatura en Hornos de Recalentamiento en APM LCA. México: Association for Iron and Steel Technology (AIST).

Wardana, A., (2005); PID-Fuzzy Controller for Grate Cooler in Cement Plant. Indonesia: Control Department, Indonesia Cement and Concrete. 